\title{
Sur l'écriture
}

\author{
Annie Ernaux
}

J]

'ai beaucoup de plaisir à être parmi vous' ${ }^{1}$ et à pouvoir tacher d'exprimer pourquoi au fond j'écris, comment j'essaie de le faire. C'est toujours très difficile, quand on écrit, de se trouver devant un public, dans une situation de conférencière. Tout d'abord parce que l'écriture, c'est la solitude, c'est ne pas voir pour quel lecteur on écrit, c'est ne pas imaginer strictement aucune personne devant soi et ne pas avoir non plus à justifier sa façon d'écrire. Cela se passe dans une sorte d'ajustement entre un désir profond au moment où on écrit, celui de faire quelque chose - je vais en parler plus loin - et les mots. C'est ce jeu perpétuel entre le désir et les mots, qui est au centre du processus d'écriture, extrêmement je répète solitaire. Alors que de se trouver dans cette situation devant vous, c'est immanquablement produire une sorte de discours, de paratexte autour de ce que l'on écrit. Quelque chose qui, d'une certaine manière, est trop signifiant, trop réducteur aussi, qui, finalement est une sorte de masque posé, entreposé, entre le lecteur et le livre. Mais, enfin, j’accepte tout de même ce risque de parler de ce que je fais, de ce que je cherche à faire depuis que j'écris.

Il y a eu, au départ, ce sentiment que la vie n'était pas suffisante. Je parle de l'époque où j'avais à peu près votre âge, c'est-à-dire entre dix-huit et vingt-deux ans, ce sentiment que vivre n'était pas suffisant, que l'écriture serait une sorte d'accomplissement, de plus d'existence. C'est ça qu'il y a eu dans mon désir d'écrire. J'ai écrit à l'époque ce que j’ai appelé un roman. J'avais vingt-deux ans, et, j'étais imprégnée par ce qui se faisait autour de moi. C'est normal, quand on commence d'écrire, de se situer par rapport

1 Conférence d'Annie Ernaux à l'Université York, le 20 octobre 1997. Texte révisé par l'auteur en janvier 2003. 
au paysage littéraire. L'époque où j'ai commencé d'écrire était celle du nouveau roman. Donc ce premier texte était marqué par les recherches du nouveau roman, d'autant plus que j'étais étudiante en littérature française et que j'avais l'habitude de lire ce qui sortait. Mon modèle a été le nouveau roman. Ce premier texte que j'ai envoyé à un éditeur n'a pas été retenu. D'une certaine façon j'en suis très contente parce que, si j'avais été publiée, peut-être n'aurais-je pas découvert finalement ce désir profond qui a alimenté ensuite tout ce que j'ai fait. Tout à l'heure, j'ai dit que l'écriture m'apparaissait comme un plus de l'existence, une sorte de valeur suprême. Mais ensuite j'ai eu un autre sentiment : ce qui m'arrive à moi, ce que j'ai vécu, n'appartient qu'à moi. Je porte quelque chose, il y a quelque chose dans ma vie, quelque chose qu'il faut mettre au jour. Et, à ce moment-là, mon désir a été de mettre au jour quelque chose que je n'avais au fond pas lu nulle part, qui était le sentiment d'une déchirure sociale entre mon milieu d'origine et le monde où j'étais entrée. J'étais passée, en effet, d'un milieu populaire très fortement marqué par les difficultés économiques, par une absence de culture (au sens où on entend le langage, le beau langage, l'habitude de fréquenter les théâtres, de lire la littérature considérée comme telle et non les romans feuilletons), à un autre monde, celui des études universitaires qui m'avait conduite à devenir moi-même professeur à vingt-six ans. Cette sensation d'avoir quelque chose à, non seulement à dire, mais à creuser, m'a conduite à écrire un livre sur cette déchirure sociale et à emprunter la forme romanesque. Ce premier livre c'est Les armoires vides.

Ce livre est pour moi vraiment le premier, le plus important d'une certaine façon. J'y considère l'écriture comme une interrogation à travers une forme littéraire. Ca c'est très important. Je veux dire que l'écriture n'est pas la psychanalyse, mais conduit à une interrogation sur la vie, en l'occurrence ma vie à moi, mais parler de sa vie à soi c'est aussi le plus souvent parler des autres. Au début je ne m'en rendais pas compte, à vrai dire : je pensais que ce qui m'était arrivé n'était arrivé qu’à moi, mais je me suis aperçue ultérieurement que, quand on descendait très loin dans son propre moi, dans son propre cheminement, qu'on essayait d'aller très loin, on rencontrait la vie des autres.

J'ai continué dans mes livres suivants à aller dans ce sens d'une quête de la réalité. Je m'explique : il y a une somme de vécus, le vécu de la déchirure sociale, mais aussi le vécu spécifiquement de femme, et tout simplement le vécu au jour le jour, quand vous prenez le métro, vous rencontrez des gens. Et l'écriture est un moyen de partir du vécu, mais d'aller vers une réalité autre. Le vécu quand on le vit, n'a pas de sens, n'a

\section{LittéRéalité}


pas de forme, on l'éprouve. Et l'écriture va permettre de dégager un sens, de dégager des choses que simplement en vivant on ne voit pas, on n'aperçoit pas.

Cette quête du réel qui me semble aller de mon premier livre jusqu'au dernier prend, bien sûr, des formes différentes. Mes trois premiers livres peuvent s'intituler des romans: Les armoires vides, Ce quils disent ou rien et La femme gelée ont une forme romanesque. Qu'est-ce que j'entends par là? D'abord j'ai mis l'indication roman, et puis, j'ai créé des personnages, une fille de quinze ans par exemple, dans ce qu'ils disent ou rien. Au moment où j'écris j'ai plus de trente ans, donc le « je " qui est utilisé dans le roman et celui de l'écrivain ne sont pas identiques, le " je » de l'héroïne est distinct de celui de l'auteur. Cependant dans La femme gelée, j'emploie le " je ", mais avec une grande indécision, puisque je ne donne pas de nom à celle qui dit « je » et on peut croire que c'est l'auteur qui parle. À partir de mon quatrième livre jusqu'à maintenant, alors là, j’ai abandonné la forme romanesque pour utiliser d'autres formes qu'on peut appeler autobiographiques, bien que ce terme-là me gène. En effet, il ne s'agit pas de raconter ma vie, comme on dit. L'autobiographie ressemble au modèle romanesque. On part, par exemple, de son enfance et on arrive au moment où l'on écrit. Ou bien on prend une période de sa vie, dont on raconte tous les événements. Pour moi, il ne s'agit pas de cela, il s'agit de rechercher à partir d'un sentiment, d'une situation personnelle, de trouver une forme. Cette forme varie suivant les livres. Il y a la forme du journal, la forme, je dirais, du récit mais, avec une analyse sociologique, historique etc. Je vais entrer plus loin dans les détails et vous lire des passages pour vous éclairer.

En attendant, je voudrais dire tout de même, quelque chose d'important. Ne croyez pas qu'écrire soit une simple opération intellectuelle : un beau jour on décide d'écrire, ensuite on continue et l'on en fait un métier d'une certaine façon. J'ai un autre métier : je suis professeur. Je n'ai pas voulu abandonner ce métier, afin d'avoir la liberté entière d'écrire ce que je veux, sans me dire que je devrai gagner ma vie avec mes livres. Non, écrire part de l'émotion, d'une sensation suffisamment forte pour provoquer l'écriture, d'une recherche de quelque chose par l'écriture. Je vous ai parlé du premier livre Les armoires vides, sur cette déchirure sociale. À un moment il y a eu quelque chose d'extrêmement fort, une sensation très forte, née en étant professeur dans des classes dont les enfants étaient d'origine populaire et, voyant ces enfants devant moi après des années d'études abstraites, ces élèves à qui j'enseignais le français, le bon français. D'un seul coup a grandi le désir de me retourner vers mon propre parcours d'enfant de classe populaire devenue prof. À l'origine de 
La femme gelée il y a également un sentiment, émotion plutôt. (L'émotion c'est quelque chose qui vient par bouffées violentes.) La sensation, à trentecinq ans, d'avoir quelque chose dans ma vie qui avait été gelé. "La femme gelée » est une femme arrêtée, figée. Ce qui avait été figé, c'était une forme de liberté, c'étaient des aspirations, des désirs qui avaient dû être réprimés. À partir de cette sensation, alors que j'écrivais en pleine période de théories féministes, j'ai tourné le dos à toute théorie et décidé de partir de l'expérience. C'est-à-dire de ne pas plaquer du tout une théorie sur mon expérience de femme, mais de retrouver, par des images, cet itinéraire de femme, de la petite fille très ouverte sur le monde et de voir comment, peu à peu, les désirs sont canalisés. Pas de théorie mais l'interrogation de mon existence.

Je passerai maintenant à un livre qui était très important pour moi, parce qu'il a inauguré un changement de forme, comme je vous disais tout à l'heure. Ce livre est La place. L'émotion au centre de ce livre ressortit sans doute à l'un des chocs les plus importants qui soient, c'est-à-dire la mort d'un père. La mort de mon père a eu lieu quand je venais d'être reçue au concours qui permet d'être professeur en France, le CAPES. Sensation violente alors que non seulement je perdais quelqu'un de cher, mais avec lui un monde qui avait été le mien, mon monde d'origine, et que ce monde allait disparaître de ma propre vie. Je devais faire quelque chose de cela. Dans La place je raconte le processus d'écriture car ça aussi, c'est un désir que j'ai, montrer comment on passe de la vie à l'écriture, et dans presque tous mes livres suivants j'explique comment, de la sensation et de la vie, on passe à l'écriture. Je vais lire ce passage qui vous donnera une idée précise de la façon dont j'écris et comment j'envisage l'écriture.

J'ai passé les épreuves pratiques du CAPES dans un lycée de Lyon à la Croix Rousse. Un lycée neuf avec des plantes vertes dans la partie réservée à l'administration et au corps enseignant, une bibliothèque au sol en moquette de sable. J'ai attendu là qu'on vienne me chercher pour faire mon cours, objet de l'épreuve, devant l'inspecteur et deux assesseurs, des profs de lettres très confirmés. Une femme corrigeait des copies avec hauteur, sans hésiter. Il suffisait de franchir correctement l'heure suivante pour être autorisée à faire comme elle toute ma vie. Devant une classe de première, des matheux, j'ai expliqué vingt-cinq lignes - il fallait les numéroter - du Père Goriot de Balzac. "Vous les avez traînés, vos élèves ", m’a reproché l'inspecteur ensuite, dans le bureau du proviseur. Il était assis entre les deux assesseurs, un homme et une fermme myope avec des chaussures roses. Moi en face. Pendant un quart d'heure, il a mélangé critiques, éloges, conseilles, et jécoutais 
à peine, me demandant si tout cela signifiait que j'étais reçue. D'un seul coup, d'un même élan, ils se sont levés tous trois, l'air grave. Je me suis levée aussi, précipitamment. L'inspecteur ma tendu la main, puis en me regardant bien en face: "Madame, je vous félicite". Les autres ont répété : "je vous félicite " et mont serré la main, mais la femme avec un sourire.

Je n'ai pas cessé de penser à cette cérémonie jusqu'à l'arrêt de bus, avec colère et une espèce de honte. Le soir même, j'ai écrit à mes parents que j'étais professeur «titulaire ". Mon père m'a répondu qu'ils étaient très contents pour moi.

Mon père est mort deux mois après, jour pour jour. Il avait soixante-sept ans et tenait avec ma mère un café-alimentation dans un quartier tranquille non loin de la gare, à Y (Seine Maritime). Il comptait se retirer dans un an. Souvent, durant quelques secondes, je ne sais plus si la scène du lycée de Lyon a eu lieu avant ou après, si le mois d'avril venteux où je me vois attendre un bus à la Croix Rousse doit précéder ou suivre le mois de juin étouffant de sa mort.

Je décris ensuite la mort de mon père. C'est un passage que je ne lirai pas, mais il s'agit d'une description mettant l'accent sur la différence des rites du décès en milieux populaire et des rites en milieu bourgeois, dans les années soixante.

Dans le train du retour, le dimanche, j'essayais d'amuser mon fils pour qu'il se tienne tranquille, les voyageurs de première n'aiment pas le bruit et les enfants qui bougent. D'un seul coup, avec stupeur, "maintenant, je suis vraiment une bourgeoise", et, "il est trop tard".

Plus tard, au cours de l'été, en attendant mon premier poste, "il faudra que j'explique tout cela". Je voulais dire, écrire au sujet de mon père, sa vie et cette distance venue à l'adolescence entre lui et moi. Une distance de classe, mais particulière, qui n'a pas de nom. Comme de l'amour séparé.

Par la suite, jai commencé un roman dont il était le personnage principal. Sensation de dégoût au milieu du récit.

Depuis peu, je sais que le roman est impossible. Pour rendre compte d'une vie soumise à la nécessité, je n'ai pas le droit de prendre d'abord le parti de l'art, ni de chercher à faire quelque chose de "passionnant " ou d' émouvant". Je rassemblerai les paroles, les gestes, les goûts de mon père, les faits marquants de sa vie, tous les signes objectifs d'une existence que j'ai aussi partagée. 
Aucune poésie du souvenir, pas de dérisions jubilantes. L'écriture plate me vient naturellement, celle-là même que jutilisais en écrivant autre fois à mes parents pour leur dire les nouvelles essentielles.

Il s'agit, non pas, comme vous le voyez, d'écrire un roman sur mon père, ni de raconter les souvenirs personnels. Rien de tout cela, c'est une approche d'une réalité, de cette réalité que j'ai perçue, confusément, à travers mon enfance, mon adolescence et qui va avoir besoin, pour être mise au jour, d'une sorte d'approche objective. Sur quoi est fondée cette approche objective? C'est remettre dans le contexte historique, géographique. C'est également relater ce que j'appelle « la culture » de mon père, mais qui était aussi ma propre culture d'origine, culture qui se fonde sur des gestes, des coutumes. Pour " remonter " ce monde, j'utilise des paroles entendues, des paroles proférées par mes parents, par les gens autour, également des photos qui sont des sortes de preuves objectives d'une époque, d'un moment dans la vie de quelqu'un. Il faut faire ressentir des lois par le concret, par le détail. C'est comme ça que je fonctionne dans l'écriture; tout ce qu'il y a de plus matériel, de plus concret peut donner accès à une réalité abstraite, à un sentiment de réalité abstraite. Écrire aussi sans aucune émotion, même si l'émotion est au départ, voyez, mais ensuite, ne pas manifester cette émotion dans l'écriture. Je vais prendre des exemples de cette méthode, de ce désir que j'ai d'atteindre quelque chose de cette façon là (désir plutôt que méthode, parce que je n’ai pas au fond, je ne peux pas dire quand je commence d'écrire que j'ai une méthode). Mais on peut dire que ça fonctionne comme ça pour moi. Par exemple, pour parler de mon père je relève ce qui est de l'ordre de l'histoire, de l'idéologie et les gestes, coutumes. Je vais lire un passage :

"L'histoire commence quelques mois avant le vingtième siècle, dans un village du pays de Caux, à vingt-cinq kilomètres de la mer. Ceux qui n'avaient pas de terre, se louaient, chez les gros fermiers de la région." "

J'emploie des mots qui ont un sens très fort : "se louaient ", ça dit tout. Les hommes qui navaient pas de terre, se donnaient eux-mêmes en quelque sorte, c'était leur personne, ils appelaient ça " se louer ". Pour donner tout le sens à cette expression j'utilise les italiques. Ces italiques sont là le signe que le sens est à prendre au pied de la lettre d'une certaine façon et contient toute la condition sociale.

"Ceux qui n'avaient pas de terre se louaient chez les gros fermiers de la région. Mon grand-père travaillait dans une ferme comme charretier. L'été, il faisait aussi les foins, la moisson. Il n'a rien fait d'autre de toute sa vie, dès l'âge de huit ans. Le samedi soir, il rapportait à sa femme toute sa paye 
et elle lui donnait son dimanche pour qu'il aille jouer aux dominos, boire son petit verre. Il rentrait saoul, encore plus sombre. Pour un rien il distribuait des coups de casquette aux enfants. C'était un homme dur, personne n'osait lui chercher des noirs. Sa femme ne riait pas tous les jours. "

Cela aussi est en italique, parce que c'est une expression très fréquente en France qui évoque la condition de la femme : elle ne riait pas tous les jours.

"Cette méchanceté était son ressort vital, sa force pour résister à la misère et croire qu'il était un homme. Ce qui le rendait violent, surtout, c'était de voir chez lui quelqu'un de la famille plongé dans un livre ou un journal. Il n'avait pas eu le temps d'apprendre à lire et à écrire. Compter, il le savait.

Chaque fois qu’on m'a parlé de lui, cela commençait par « il ne savait ni lire ni écrire " comme si sa vie et son caractère ne se comprenaient pas sans cette donnée initiale. Ma grand-mère, elle, avait appris à l'école des sœurs. Comme les autres femmes du village, elle tissait chez elle pour le compte d'une fabrique de Rouen, dans une pièce sans air recevant un jour étroit d'ouvertures allongées à peine plus larges que des meurtrières. Les étoffes ne devaient pas être abîmées par la lumière. Elle était propre sur elle et dans son ménage, qualité la plus importante au village, où les voisins surveillaient la blancheur et l'état du linge en train de sécher sur la corde et savaient si le seau de nuit était vidé tous les jours. Bien que les maisons soient isolées les unes des autres par des haies et des talus, rien n'échappait au regard des gens, ni l'heure à laquelle l'homme était rentré du bistrot, ni la semaine où les serviettes hygiéniques auraient dû se balancer au vent.

Le signe de croix sur le pain, la messe, les pâques. Comme la propreté, la religion leur donnait la dignité. Ils s'habillaient en dimanche, chantaient le Credo en même temps que les gros fermiers, mettaient des sous dans le plat. Mon père était enfant de chœur, il aimait accompagner le curé porter le viatique. Tous les hommes se découvraient sur leur passage.

Les enfants avaient toujours des vers. Pour les chasser on cousait l'intérieur de la chemise, près du nombril, une petite bourse remplie d'ail. L'hiver, du coton dans les oreilles. Quand je lis Proust ou Mauriac, je ne crois pas qu'ils évoquent le temps où mon père était enfant. Son cadre à lui, c'est le Moyen Âge. »

L'histoire de mon père est racontée dans un contexte historique et dans un contexte historique et à travers d'une objectivation des faits culturels. Je prendrai un autre exemple :

" Il reconnaissait les oiseaux à leur chant et regardait le ciel chaque soir pour savoir le temps qu'il ferait, froid et sec s'il était rouge, pluie et vent 
quand la lune était dans l'eau, c'est-à-dire immergée dans les nuages. Tous les après-midi il filait à son jardin, toujours net. Avoir un jardin sale, aux légumes mal soignés, indiquait un laisser-aller de mauvais aloi, comme se négliger sur sa personne ou trop boire. C'était perdre de la notion du temps, celui où les espèces doivent se mettre en terre, le souci de se que penseraient les autres. Parfois des ivrognes notoires se rachetaient par un beau jardin cultivé entre deux cuites. Quand mon père n'avait pas réussi des poireaux ou n'importe quoi d'autre, il y avait du désespoir en lui. À la tombée du jour, il vidait le seau de nuit dans la dernière rangée ouverte par la bêche, furieux s'il découvrait, en le déversant, des vieux bas et des stylos billes que j'y avais jetés par paresse de descendre jusqu'à la poubelle.

Pour manger, il ne se servait que de son Opinel. Il coupait le pain en petits cubes, disposés près de son assiette pour y piquer des bouts de fromage, de charcuterie, et saucer. Me voir laisser de la nourriture dans l'assiette lui faisait deuil. On aurait pu ranger la sienne sans la laver. Le repas fini, il essuyait son couteau contre son bleu. S’il avait mangé de hareng, il l'enfouissait dans la terre pour lui enlever l'odeur. Jusqu'à la fin des années cinquante il a mangé de la soupe le matin, après il s'est mis au café au lait, avec réticence, comme s'il sacrifiait à une délicatesse féminine. Il le buvait cuillère par cuillère, en aspirant comme de la soupe. À cinq heures, il se faisait sa collation, des œufs, des radis, des pommes cuites et se contentait le soir d'un potage. La mayonnaise, des sauces compliquées, les gâteaux, le dégoûtaient.

Ce portrait, j'aurais pu le faire autrefois, en rédaction, à l'école, si la description de ce que je connaissais n'avait pas été interdite. Un jour, une fille, en classe CM2, a fait s'envoler son cahier par un splendide atchoum. La maîtresse au tableau s'est retournée "Distingué, vraiment. »

Dans d'autres livres j'écris de la même façon concrète, objective, même sur un sujet très différent, comme la passion. Il y a quelques années, la passion a été une expérience violente pour moi et aussitôt après, il y a eu ce désir-là, non pas d'écrire un roman sur la passion, non plus de raconter cette histoire, mais d'essayer de répondre à ceci : qu'est-ce qui m'a traversé, qu'est-ce que c'est qu'une passion? Sans d'ailleurs que j'y pense vraiment, ma façon d'écrire a été sensiblement la même que dans La Place. J'ai recensé ce que j'appelle tous les signes concrets, objectifs, de la passion.

« À partir du mois de septembre l'année dernière, je n'ai plus rien fait d'autres qu'attendre un homme : qu'il me téléphone et qu'il vienne chez moi. J'allais au supermarché, au cinéma, je portais des vêtements au pressing, je lisais, je corrigeais des copies, j’agissais exactement comme avant, mais sans une longue accoutumance de ces actes cela m'aurait été 
impossible, sauf au prix d'un effort effrayant. C'est surtout en parlant que j'avais l'impression de vivre sur ma lancée.Les mots et les phrases, le rire même se formaient dans ma bouche sans participation réelle de ma réflexion ou de ma volonté. Je n'ai plus d'ailleurs qu'un souvenir vague de mes activités, des films que j'ai vus, des gens que j'ai rencontrés. L'ensemble de ma conduite était factice. Les seules actions où j'engageais ma volonté, mon désir et quelque chose qui doit être l'intelligence humaine (prévoir, évaluer le pour et le contre, les conséquences) avaient toutes un lien avec cet homme :

lire dans le journal les articles sur son pays (il était étranger), choisir des toilettes et des maquillages, lui écrire des lettres, changer les draps du lit et mettre les fleurs dans la chambre, noter ce que je ne devais pas oublier de lui dire la prochaine fois, qui était susceptible de l'intéresser, acheter du whisky, des fruits, diverses petites nourritures pour la soirée ensemble, imaginer dans quelle pièce nous ferions l'amour à son arrivée.

Je n'avais pas d'autre avenir que le prochain coup de téléphone fixant un rendez-vous. J'essayais de sortir le moins possible en dehors de mes obligations professionnelles - dont il avait les horaires - craignant toujours de manquer un appel de lui pendant mon absence. J'évitais aussi d'utiliser l'aspirateur ou le sèche-cheveux qui m'auraient empêché d'entendre la sonnerie. Celle-ci me ravageait d'un espoir qui ne durait souvent que le temps de saisir lentement l'appareil et de dire "allô ». En découvrant que ce n'était pas lui, je tombais dans une telle déception que je prenais en horreur la personne ou bout de fil. Dès que j'entendais la voix de A., mon attente indéfinie, douloureuse, jalouse évidemment, s'anéantissait si vite que j'avais l'impression d'avoir été folle et de redevenir subitement normale. J'étais frappée par l'insignifiance, au fond, de cette voix et l'importance démesurée qu'elle avait dans ma vie.

Une fois prête, maquillée, coiffée, la maison rangée, j'étais s'il m'en restait du temps, incapable de lire ou de corriger des copies. D'une certaine façon si je ne voulais pas détourner mon esprit de l'autre chose que l'attente de A : ne pas gâcher celle-ci. Souvent j'écrivais sur une feuille la date, l'heure et « il va venir " avec d'autres phrases, des craintes qu'il ne vienne pas, qu'il ait moins de désir. Le soir je reprenais cette feuille, il est venu, notant en désordre les détails de cette rencontre. Puis je regardais, hébétée, la feuille gribouillée avec les deux paragraphes écrits avant et après qui se lisaient à la suite, sans rupture. Entre les deux il y avait eu des paroles, des gestes, qui rendaient tout le reste dérisoire, y compris l'écriture par laquelle j'essayais de les fixer. Un espace de temps, délimité par deux bruits de voiture, sa $\mathrm{R}$ vingt-cinq, freinant, redémarrant, où j'étais sûre qu'il n'y avait 
jamais rien eu de plus important dans ma vie, ni d'avoir des enfants, ni réussir des concours, ni voyager loin, que cela, être au lit avec cet homme au milieu de l'après midi. »

Tout cela est du concret, même les pensées peuvent être considérées, objectivées, considérées comme des faits concrets.

"Durant cette période je n'ai pas écouté une seule fois de la musique classique, je préférais les chansons. Les plus sentimentales, auxquelles je ne prêtais aucune attention avant, me bouleversaient. Elles disaient sans détours ni distance, l'absolu de la passion, et aussi son universalité. En entendant Sylvie Vartan chanter alors "C'est fatal, animal ", j'étais sûre de ne pas être la seule à éprouver cela. Les chansons accompagnaient et légitimaient ce que j'étais en train de vivre.

Il me prenait l'envie de voir sans délai des films dont j'étais persuadée qu'ils contenaient mon histoire, très déçue si lorsqu'il était ancien, on ne le jouait nulle part, comme « L'empire des sens » d'Oshima.

Un après-midi où il était là, j’ai brûlé le tapis du living jusqu'à la trame en posant dessus une cafetière brûlante. Cela m'était indifférant. Même, à chaque fois que j'apercevais cette remarque, j'étais heureuse en me rappelant cet après-midi avec lui.

Tout ce temps, j'ai eu l'impression de vivre ma passion sur le mode romanesque, mais je ne sais pas, maintenant, sur quel mode je l'écris : si c'est celui du témoignage, voire de la confidence telle qu'elle se pratique dans les journaux féminins, celui du manifeste ou du procès-verbal, ou même du commentaire du texte.

Je ne fais pas le récit d'une liaison, je ne raconte pas une histoire (qui m'échappe pour la moitié) avec une chronologie précise "il vient le 11 novembre " ou approximative, " des semaines passèrent ". Il n'y en avait pas pour moi dans cette relation, je ne connaissais que la présence ou l'absence. J'accumule seulement les signes d'une passion oscillant sans cesse entre « toujours » et « un jour », comme si cet inventaire allait me permettre d'atteindre la réalité de cette passion. Il n'y a naturellement ici, dans l'énumération et la description des faits, ni ironie, ni dérision, qui sont des façons de raconter les choses aux autres ou à soi-même après les avoir vécues, non de les éprouver sur le moment.

Quant à l'origine de ma passion, je n'ai pas l'intention de la chercher dans mon histoire lointaine, celle que me ferait reconstituer un psychanalyste, ou récente, ni dans les modèles culturels du sentiment qui m'ont influencée depuis l'enfance (Autant en emporte le vent, Phèdre, ou les chansons de Piaf sont aussi décisifs que le complexe d'Edipe). Je ne veux pas expliquer ma passion - cela reviendrait à la considérer comme 
une erreur ou un désordre dont il faut se justifier - mais simplement l'exposer.

Les seules données, peut-être, à prendre en compte seraient matérielles, le temps et la liberté dont j'ai pu disposer pour vivre cela. »

Vous avez peut-être remarqué la présence de détails qui peuvent apparaître comme triviaux. À mes yeux, il n'y a pas de hiérarchie dans les objets qui peuvent entrer dans l'écriture, puisque, si l'écriture, comme je la considère, est une quête de la réalité, tout a une importance, tout a un sens. Aussi bien la cafetière que la chanson de Sylvie Vartan que l'on écoute. Je ne confere pas une dignité ou une indignité aux objets en général, à tout ce qui peut être écrit. Il n'y a pas de choses belles par elles-mêmes ou certains sentiments. Il n'y a tout simplement que la réalité et l'écriture et la recherche de celle-ci.

Pour des étudiants en lettres, cette question se pose "quelle est votre voix narrative à l'intérieur des textes »? Depuis le début j'utilise le « je », mais le statut de ce « je » a varié depuis mes premiers livres jusqu’au dernier. Au début, dans les premiers livres, le " je » est celui à la fois du personnage et celui de la narratrice, donc la voix varie. Dans Les armoires vide, le « je " exprime les affects de la petite fille, mais à d'autres moments c'est la narratrice qui analyse. Les deux " je " se confondent à la lecture. Ultérieurement, à partir de La place, le « je " va renvoyer à l'auteur, c'est la personne de l'auteur, et en même temps, c'est une voix le plus souvent impersonnelle : un « je » qui est vidé d'affect. Vous avez pu remarquer, par exemple dans Passion simple, que ce " je » est une voix sans expression de sentiment, d'émotion, simplement un " je » qui dit les faits. Je considère de plus en plus le « je » que j'emploie comme " un signe vide ». Une sorte de lieu où peut passer une expérience, mais qui ne renvoie pas à une personne explicitement. Ce " je » que j'emploie oblige, d'une certaine manière, le lecteur à prendre sa place dans le texte. Quand on utilise " je ", le lecteur au moment où il lit, lit « je » aussi, et cela le renvoie à sa propre expérience. Là, il y a deux réactions possibles : soit il dit " non, non, cela ne me concerne pas, je ne suis pas du tout impliqué ", et le lecteur s'éloigne du texte, ou bien il s'en ressent très proche. Le « je » rapproche le plus l'écriture et la vie du lecteur. Il y a une sorte de circularité : j'essaie de saisir, de ressaisir la vie au moyen de l'écriture, et aussi d'une certaine manière il y a dans cette démarche le désir que le lecteur se projette avec sa propre vie dans le texte, d'établir une sorte de circularité vie-écriture-vie. Pour moi la littérature n'est pas un jeu, la littérature n'a pas seulement une fonction esthétique, elle a une fonction de la vie. Les livres que j'ai aimés ont eu une grande importance dans ma formation, ma structuration consciente et 
inconsciente, et je crois que c'est cela à quoi doit tendre l'écriture, avoir un rôle dans la vie des gens. Bien entendu, ce rôle-là ne peut être atteint que par des exigences très fortes en écrivant, c'est-à-dire qu'on ne peut pas renoncer à la fonction esthétique, c'est évident, mais il faut se dire que cette esthétique est au service d'une action dans le monde.

Il reste quelque dix minutes, j'aimerais bien que vous posiez des questions, n'importe lesquelles, car j'ai juste évoqué ici quelques directions.

\section{Questions}

Si j'ai bien compris, Madame Ernaux, l'écriture pour vous naît d'une expérience personnelle très profonde, viscérale et sociale?

Oui, c'est le sentiment d'une spécificité de mon expérience, qui m’a conduite à la mettre au jour, parce qu'elle n'avait pas, à mon sens, été dite. En évoquant cette réalité du monde social, cette déchirure sociale dont j'ai parlé dans mon premier livre - qui a suscité dans le champ littéraire français un grand intérêt, "voilà une chose qu'on n'a pas lue ». C'est aussi l'existence, la légitimité de ce premier monde, le monde dominé. Également, je retrouve par les mots une sorte de langue matérielle, car je suis passée du monde où les mots et les choses étaient, d'une certaine manière violente, collés ensemble, au monde de l'abstraction, au monde des études où on est très éloigné des choses, n'est-ce pas? C'est le monde où on ne travaille pas de ses mains. C'est un où tout se transforme en concept, en idée, c'est certain, nous sommes là pour ça, à l'université, et ça commence dès l'école. J'ai eu ce sentiment qu'il fallait avec les mots abstraits, comme dit Jean Genet "la langue de l'ennemie " aller en retrouver le monde premier, pour donner consistance à ce monde où l'on travaille de ses mains. Même quand je ne parle pas de ce monde, par exemple comme dans Je ne suis pas sortie de ma nuit, mon dernier texte sur ma mère, je ne vois que les détails matériels concrets comme les chaussettes brunes qu'on lui met. C'est comme ça que je peux saisir la douleur. Ce n'est pas de l'abstrait, c'est la force des choses. C'est donner aux mots qui sont forcément quelque chose de léger et avec lesquels on peut jouer, leur donner la force qu'ont les choses quand on ne parle même pas, qu'on est confronté aux expériences les plus fortes, celles de la mort, de la maladie, de l'amour ou simplement de la peur. Je veux dire la vie au sens le plus fort. 
Je dirais que faire l'apprentissage de la vie, c'est perdre une certaine innocence, pureté. En vous lisant, Madame Ernaux, jai le sentiment que l'écriture est aussi une sorte de salissure, quelque chose d'impur?

Oui, c'est vrai, j'ai dit que l'écriture était une chose impure. «Impure » n'a pas de notion de valeur morale pour moi. Ce mot s'oppose à "clean ", propre. Il contient l'idée de descendre. Pour moi l'image de l'écriture c'est de descendre dans des choses troubles, vraiment très troubles, et se trouver confronté à la difficulté de les dire, même à la transgression de les dire. Finalement c'est une transgression et beaucoup de choses sont inconscientes quand on écrit. Écrire est une activité qui quelquefois me terrifie. L'écriture, c'est terrifiant; on ne sait plus où on va. Mais peut-être dans " impure ", il y a l'idée de faute, de faute sexuelle, je ne sais pas, l'idée qu'écrire ce n'est pas bien. Il y a un passage dans Passion simple où j'évoque l'écriture comme quelque chose de caché, ressemblant à ces mots obscènes que l'on écrit parfois. Voici ce passage :

"Et si encore devant les feuilles couvertes de mon écriture entourée illisible sauf pour moi je peux croire quil s'agit de quelque chose de privé, de presque en fontane portant pas une conséquence, comme les déclarations d'amour et phrases obscènes que j'inscrivais en classe à l'intérieur de mes protège-cahiers, et tout ce qu'on peut écrire tranquillement, impunément tant qu'on est sûr que personne ne le verra. Quand je commence à taper ce texte à machine qui n’apparâtra dans les caractères publics, mon innocence sera finie. »

Vous voyez impureté, innocence, il y a peut-être là, quelque chose, je ne sais pas. Vous savez, je ne pense pas à tout quand j'écris. Je ne pense pas au lecteur qui lira cela. Le lecteur est absent.

Madame Ernaux, vous êtes maintenant une écrivaine bien connue, célèbre. Comment la publication a changé votre vie?

Ce qui change d'être publiée? Eh bien, justement, je me le demande. Dans ma vie personnelle, pour la vie personnelle, je ne vois pas grande chose de changé. Je continue de vivre et l'écriture occupe une grande partie de ma vie, mais la publication, dans ma vie propre, ne change rien. Une fois qu'un livre est fait, la vie n'est pas éclaircie, contrairement à ce qu’on peut penser. C'est-à-dire, j'essaie d'atteindre une réalité, mais ma propre vie à moi, ne me parât pas éclaircie pour autant. Par contre il y a un livre. Brusquement, il y a un livre. J'ai terminé un texte, mais je ne sais pas ce que j'ai fait. Je ne sais jamais ce que j'ai fait. La publication, ça veut dire 
des gens qui lisent, et qui vous disent ceci, cela, et c'est comme ça que le livre se met à prendre corps, à avoir un sens en ce moment-là. C'est peutêtre étonnant, mais je n'ai aucune relation avec mes textes écrits, d'une certaine façon.

Vous venez de nous dire que l'écriture est la recherche de la vérité. L'écriture est donc un lieu où l'on ne peut mentir?

Oui. Dans la vie, la vie sociale nous oblige à des compromissions, à la politesse, à toutes sortes. C'est ça la civilisation. S'il y a un lieu où on doit être vrai, c'est l'extrême. C'est le lieu où justement on ne peut pas mentir d'une certaine façon. Parce que je mets cette recherche dans l'écriture, j'ai besoin de liberté, de temps. Je ne publie que tous les trois ans, quatre ans. L'écriture n'est pas un métier pour moi. Quand j'entends parler du métier d'écrivain, ça me fait toujours bizarre. Ce que je sais, c'est que, quand je commence à écrire, j'ai toujours l'impression de ne pas savoir : je n'ai pas de " métier ". C'est normal puisque je décris en partant d'un sentiment, que je ne construis pas une histoire. Donc les difficultés sont entières. Pour mon dernier livre, La honte, je suis partie d'une scène de mon enfance, après je ne savais pas. Il y avait la scène et puis quoi? Il y a eu toute cette recherche pour aller plus loin et je me suis aperçue que je devais remonter le monde de mon enfance. Comment allais-je faire pour remonter ce monde? L'écriture tout au long est un parcours, une sorte d'interrogation continuelle. C'est une entreprise longue et difficile. 